\title{
A Rare Presentation of Stauffer's Syndrome Associated with Renal Cell Carcinoma
}

\author{
Bashar Tanous $^{\mathrm{a}}$ Arwa Alsaud $^{\mathrm{a}}$ Khalid E. Mahmoud ${ }^{\mathrm{b}}$ \\ Mohamed A. Yassin ${ }^{c}$ \\ aDepartment of Internal Medicine, Hamad Medical Corporation, Doha, Qatar; ${ }^{b}$ Department \\ of Urology, Hamad Medical Corporation, Doha, Qatar; ' Department of Medical Oncology, \\ Hamad Medical Corporation, Doha, Qatar
}

\section{Keywords}

Renal cell carcinoma · Paraneoplastic syndrome · Liver disease · Cancer · Qatar

\begin{abstract}
Renal cell carcinoma (RCC) is a primary tumor of the kidneys. It is characterized by the triad of flank pain, hematuria, and a palpable flank mass. However, most RCC patients present with a paraneoplastic manifestation of the disease. Stauffer's syndrome (a non-metastatic hepatic dysfunction) is a rare paraneoplastic manifestation associated with RCC. We report the case of a 30-year-old male, obese, who presented with hematuria and was found to have a right renal mass secondary to RCC. During his hospital stay, the patient developed acute hepatic dysfunction that resolved rapidly after tumor resection.

\section{Introduction}

Renal cell carcinoma (RCC) accounts for $80-85 \%$ of all primary renal neoplasms [1]. Roughly $20 \%$ of all patient with RCC can present with paraneoplastic manifestations [2]. A paraneoplastic syndrome is defined as a constellation of signs and symptoms that accompany a malignant disease which cannot directly be attributed to tumor extension or metastasis [3]. Paraneoplastic manifestations that associate with RCC can be divided into endocrine (i.e., hypercalcemia, hypertension, polycythemia, non-metastatic hepatic dysfunction, etc.) and non-endocrine (i.e., anemia, amyloidosis, etc.) paraneoplastic manifestations [4].

The non-metastatic hepatic dysfunction known as Stauffer's syndrome was first described by Maurice H. Stauffer in 1961 [5], when he noted that patients with RCC had abnormal liver 
Fig. 1. MRI shows a large left retroperitoneal mass measuring $12.6 \times 11.2 \times 13.5 \mathrm{~cm}$ (maximum AP $\times \mathrm{TR} \times \mathrm{CC}$ dimensions) arising from the upper part of the left kidney. It is exophytic and involves the renal parenchyma, extending into the upper collecting system. It is markedly heterogenous, with extensive internal cystic non-enhancing necrotic areas. The solid components show enhancement and diffusion restriction. There are a few scattered areas of blood products/hemorrhage.

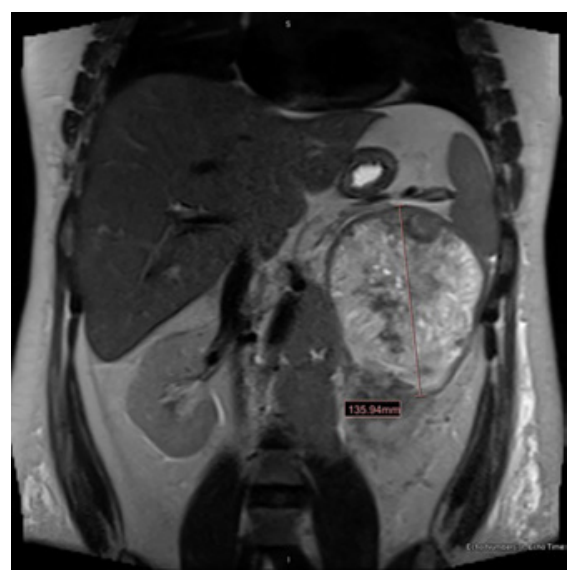

function tests in the absence of hepatic metastasis. Removal of the tumor led to a normalization of these tests. He came to the conclusion that this hepatic dysfunction was paraneoplastic in nature [5].

Stauffer's syndrome is characterized by impaired hepatic synthetic function and elevated liver enzymes. The most common laboratory finding is elevated alkaline phosphatase, seen in $90 \%$ of all cases. While elevated hyperbilirubinemia and transaminase have been found in 15 and $21 \%$ of all cases, respectively, other laboratory abnormalities are a prolongation of prothrombin time, an elevation of gamma-glutamyltransferase, erythrocyte sedimentation rate, alpha-2-globulin, and thrombocytosis [6].

Here, we report an unusual presentation of Stauffer's syndrome.

\section{Case Presentation}

A 30-year-old Pakistani male presented to our hospital complaining of a 3-day history of hematuria and left flank pain. The patient was not known to have any co-morbidities. He did not have any other associated symptoms. He had never drunk alcohol and had never smoked before.

Upon evaluation, his vital signs were normal. He had a high BMI of 34.3 (normal range: 18.5-24.9). A general physical examination revealed a palpable left flank mass confirmed by ultrasound to be a $11-\mathrm{cm}$ solid mass in his left kidney with central necrosis. Images were suggestive of RCC. Initial routine blood analyses reported a normal white blood cell count, a low hemoglobin level of $10.9 \mathrm{~g} / \mathrm{dL}$ (normal range: 13.0-17.0), serum creatinine of $121 \mu \mathrm{mol} / \mathrm{L}$ (normal range: 62-106), urea of $4.70 \mathrm{mmol} / \mathrm{L}$ (normal range: 2.76-8.07), and normal liver function tests with total bilirubin of $5 \mu \mathrm{mol} / \mathrm{L}$ (normal range: $0-21$ ), alkaline phosphatase of 73 U/L (normal range: 40-129), alanine transferase of 33 IU (normal range: 0.0-41), and aspartate transferase of 24 IU (normal range: 0.0-40).

An MRI of the abdomen with contrast (Fig. 1) showed a large left retroperitoneal mass measuring $12.6 \times 11.2 \times 13.5 \mathrm{~cm}$ (maximum anterior-posterior (AP) $\times$ transverse $(\mathrm{TR}) \times$ craniocaudal (CC) dimensions) arising from the upper part of the left kidney which was suggestive of RCC. Incidentally, the MRI showed a contiguous thrombus in the left renal vein extending into the inferior vena cava (Fig. 2, Fig 3), and markedly enlarged left para-aortic lymph nodes. Other organs such as the liver, gallbladder, and biliary tree showed no pathology. The patient underwent emergent left renal artery embolization and was planned for a radical left nephrectomy in the following couple of days. 
Fig. 2. MRI shows a large contiguous tumor thrombus extending from the left upper renal mass into the left renal vein, completely filling and distending the left renal vein, and protruding into the IVC lumen, without significant extension above the level of the renal vein within the IVC $(3.6 \mathrm{~cm}$ CC dimension, IVC thrombus).

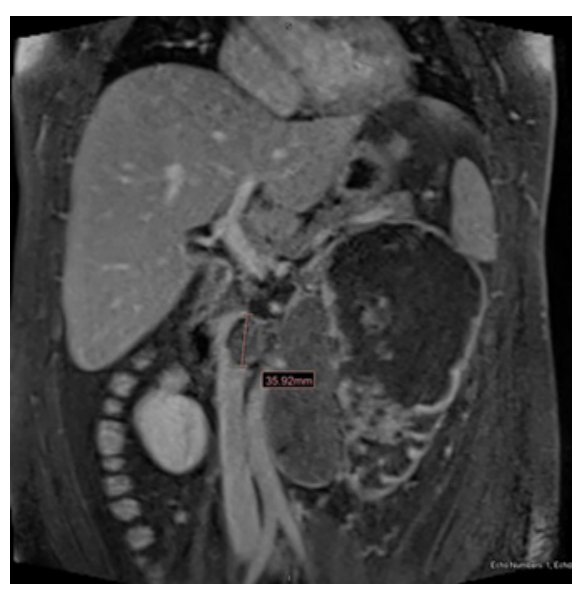

Fig. 3. FDG-PET CT shows a moderate to intense peripheral uptake (SUVmax around 10) in the known large left upper renal mass with a maximal diameter of $13 \mathrm{~cm}$. The increased uptake extends into the dilated left renal vein and can be followed to the IVC itself. A similar level of uptake is seen in two enlarged left para-aortic retroperitoneal lymph nodes $(6-7 \mathrm{~cm}$ in size).

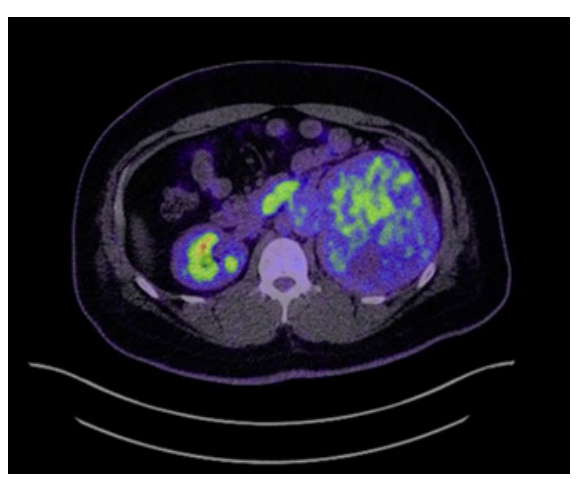

On day 5 after hospitalization, the patient developed abnormal liver function tests. There was a significant increase in alkaline phosphatase of $951 \mathrm{U} / \mathrm{L}$ (normal range: 40-129) and alanine and aspartate transferase levels of 56 IU (normal range: 0.0-41) and 324 IU (normal range: $0.0-40$ ), respectively. His coagulation profile and bilirubin level were within normal range. Our patient underwent further investigations to determine the underlying etiology of his deranged liver enzymes. Screening for viral hepatitis B and C were negative. Repeated liver function tests showed persistent elevated alkaline phosphatase levels ranging between 300 and $400 \mathrm{U} / \mathrm{L}$ (normal range: 40-129), alanine transferase was back to normal but the aspartate transferase level continued to be high ranging between 100 and 200 IU (normal range: 0.0-40). A repeated MRI of the abdomen with contrast before radical nephrectomy was not suggestive of biliary tree involvement or liver pathology. The patient underwent left radical nephrectomy plus inferior vena cava thrombectomy.

On the morning of the procedure, our patient's alkaline phosphatase level was $298 \mathrm{U} / \mathrm{L}$ (normal range: 40-129), aspartate transferase was 118 IU (normal range: 0.0-40) with a normal alanine transferase level. However, $1 \mathrm{~h}$ after the procedure, repeated labs showed that his liver function tests were back to normal: alkaline phosphatase was $60 \mathrm{U} / \mathrm{L}$ (normal range: 40-129) and aspartate transferase was 36 IU (normal range: 0.0-41). The pathology report of the left kidney revealed unclassified RCC. At follow-up, after 2 months from the procedure, the tests also showed persistently normal liver functions.

Our diagnosis of RCC was confirmed by the rapid normalization of liver functions after removing the tumor as previously described in the literature and as will be further described in the Discussion below. 


\section{Discussion}

Kidney tumors account for 3\% of all new cancer diagnoses [7]. RCC is considered to be the predominant tumor type which arises in the renal parenchyma [8]. The incidence of RCC varies from one region to the other: approximately 74,000 new cases of RCC are reported each year in the USA with almost 15,000 deaths annually from the disease [9]. The most distinguished risk factors are obesity and smoking, accounting for about 30 and $20 \%$ of all RCC cases, respectively [10].

The classical triad of RCC presentation is flank pain, hematuria, and a palpable flank mass; however, this triad is seen in $<10 \%$ of the cases. Most RCC patients present with a paraneoplastic manifestation of the disease [11]. A recent review looking at all reported cases of Stauffer's syndrome yielded $>100$ cases, most of which described the association with RCC; the incidence rate was 3-6\% among these patients [6].

Multiple theories have been proposed to try and explain the pathophysiology of Stauffer's syndrome behind this paraneoplastic manifestation. One hypothesis relates it to the high lysosomal enzyme activity which was found in the liver cells of RCC patients suggesting that the tumor is releasing these substances which have a direct effect on the liver [6]. Other hypotheses suggested hepatic amyloid deposition, generalized hepatic hypervascularity, nonspecific focal periportal inflammation, or an autoimmune phenomenon which cross-reacts with the liver protein [6]. The majority of reported cases in the literature had elevated IL-6, and an association between IL-6 and Stauffer's syndrome was suggested [6].

Most of the cases reported in the literature presented with liver impairment; and as part of their abnormal liver function test work-up, they were discovered to have RCC, while in our case, our patient presented with RCC and developed Stauffer's syndrome acutely in the hospital - which resolved completely after removing the tumor. Our case should increase physicians' awareness of this rare paraneoplastic manifestation of RCC which could be the first presentation of the disease.

\section{Conclusion}

Our case describes the acute presentation of Stauffer's syndrome developing during hospital stay and which resolved completely following tumor removal. To the best of our knowledge, such a case has not previously been described in the literature.

\section{Acknowledgment}

We would like to thank the internal medicine residency program of Hamad Medical Corporation for scientific support.

\section{Statement of Ethics}

The patient has given his written informed consent to publish his case (including publication of images). 


\section{Conflict of Interest Statement}

The authors declare no conflicts of interest.

\section{Funding Sources}

Funding was received from Qatar National Library.

\section{Author Contributions}

Bashar Tanous: writing and editing of the manuscript. Arwa Alsaud: editing of the manuscript. Khalid E. Mahmoud provided the radiological images. Mohamed A. Yassin: editing of the manuscript.

\section{References}

1 Alsamman MA, Draper D. Nonmetastatic renal cell carcinoma presenting with persistent cough: case report with literature review. Avicenna J Med. 2019;9(4):160-3.

2 Gold PJ, Fefer A, Thompson JA. Paraneoplastic manifestations of renal cell carcinoma. Semin Urol Oncol. 1996; 14(4):216-22.

3 Ikuerowo SO, Ojewuyi OO, Omisanjo OA, Abolarinwa AA, Bioku MJ, Doherty AF. Paraneoplastic syndromes and oncological outcomes in renal cancer. Niger J Clin Pract. 2019 Sep 1;22(9):1271-5.

4 Palapattu GS, Kristo B, Rajfer J. Paraneoplastic syndromes in urologic malignancy: the many faces of renal cell carcinoma. Rev Urol. 2002;4(4):163-70.

5 Morla D, Alazemi S, Lichtstein D. Stauffer's syndrome variant with cholestatic jaundice: a case report. J Gen Intern Med. 2006;21(7):C11-3.

6 Sharma N, Darr U, Darr A, Sood G. Stauffer syndrome: a comprehensive review of the icteric variant of the syndrome. Cureus. 2019 Oct;11(10):e6032.

7 Mindrup SR, Pierre JS, Dahmoush L, Konety BR. The prevalence of renal cell carcinoma diagnosed at autopsy. BJU Int. 2005 Jan;95(1):31-3.

8 Chow WH, Dong LM, Devesa SS. Epidemiology and risk factors for kidney cancer. Nat Rev Urol. 2010;7(5): 245-57.

9 Siegel RL, Miller KD, Jemal A. Cancer statistics, 2020. CA Cancer J Clin. 2020 Jan;70(1):7-30.

10 Lipworth L, Tarone RE, McLaughlin JK. The epidemiology of renal cell carcinoma. J Urol. 2006;176(6 Pt 1): 2353-8.

11 Ateş I, Kaplan M, Yilmaz N. A Stauffer's syndrome variant associated with renal cell carcinoma and thrombocytopenia. Afr J Urol. 2015 Dec 1;21(4):217-9. 